\author{
MITSUBISHI ELECTRIC RESEARCH LABORATORIES \\ http://www.merl.com
}

\title{
Implementing Backscatter Radio for Wireless Sensor Networks
}

\author{
Giovanni Vannucci, Aggelos Bletsas, Darren Leigh
}

TR2007-088 August 2008

\begin{abstract}
We provide an overview of our experimental system, testing in practice a sensor communicating through backscatter at a range of approximately 15 meters indoors, with $5 \mathrm{~mW}$ transmission power at 10 bits per second. Our system is designed for simultaneous reception of signals continuously radio-backscattered from several ultra low-cost sensors. This work highlights the idiosyncracies of the backscatter channel and presents a proof-of-concept demonstration of backscatter radio for wireless sensor networks, especially when low bit-rate, ultra low-cost sensors are required.
\end{abstract}

PIMRC 2007

This work may not be copied or reproduced in whole or in part for any commercial purpose. Permission to copy in whole or in part without payment of fee is granted for nonprofit educational and research purposes provided that all such whole or partial copies include the following: a notice that such copying is by permission of Mitsubishi Electric Research Laboratories, Inc.; an acknowledgment of the authors and individual contributions to the work; and all applicable portions of the copyright notice. Copying, reproduction, or republishing for any other purpose shall require a license with payment of fee to Mitsubishi Electric Research Laboratories, Inc. All rights reserved. 



\section{IMPLEMENTING BACKSCATTER RADIO FOR WIRELESS SENSOR NETWORKS}

\author{
Giovanni Vannucci \\ Mitsubishi Electric Research \\ Laboratories, Cambridge USA
}

\author{
Aggelos Bletsas \\ RadioCommunications Lab (RCL) \\ Aristotle University of Thessaloniki, Greece*
}

\author{
Darren Leigh \\ Mitsubishi Electric Research \\ Laboratories, Cambridge USA
}

\begin{abstract}
We provide an overview of our experimental system, testing in practice a sensor communicating through backscatter at a range of approximately 15 meters indoors, with $5 \mathrm{~mW}$ transmission power at 10 bits per second. Our system is designed for simultaneous reception of signals continuously radio-backscattered from several ultra low-cost sensors. This work highlights the idiosyncracies of the backscatter channel and presents a proofof-concept demonstration of backscatter radio for wireless sensor networks, especially when low bit-rate, ultra low-cost sensors are required.
\end{abstract}

\section{INTRODUCTION}

The technique of radio backscatter is commonly used in RFID (Radio Frequency IDentification) systems. Radio backscatter communication is an attractive solution in such systems because various involved tags do not need to actively transmit any radio signal. Instead, they simply reflect a radio signal transmitted by an interrogator, and modulate the reflection by controlling their own reflection coefficient [1].

This leads to low-complexity, low-power and low-cost implementations for each involved tag. In this work, we attempt to exploit those properties of backscatter radio in low-cost, energy efficient wireless sensor networks. For example, we envision several hundred sensors that continually report the values of environmental parameters (e.g. temperature, humidity or pollutant concentration) to a central hub, using backscatter radio.

Fig. 1 shows block diagrams for the hub and one of the sensors, as implemented in this work. The goal was to make sensors as simple and low-cost as possible while concentrating the more complex functionality into the central hub, shared among all sensors. Accordingly, each sensor implements backscatter communication by simply switching on and off the impedance connected to the antenna port. Thus, energy consumption at each sensor is required just to control a switch transistor, while energy-demanding communication functions (e.g. signal power amplification) or complex RF-front ends at each sensor are completely omitted. The central hub includes a transmitter that transmits an unmodulated RF carrier out of the transmitting antenna (Tx Ant). The receiving antenna (Rx Ant) picks up the signals reflected (backscattered) back by each sensor antenna.

Implementing backscatter radio for wireless sensor networks, as envisioned and implemented in this work, becomes challenging due to a) the absence of radio receiver at each sensor (and thus, wireless communication is unidirectional), b) the

${ }^{*}$ This work was done while Dr. Bletsas was a post-doctoral fellow at Mitsubishi Electric Research Laboratories (MERL), Cambridge MA, USA. unsuitability of traditional radio techniques for multi-path mitigation (as we show in this work) and c) the need for a flexible experimental setup supporting several sensors, easy to use, test and modify. This work discusses the methods used to address the above challenges and presents a proof-of-concept demonstration of backscatter radio for wireless sensor networks.

We present the basic system approach and relevant equations in section II. We briefly present an overview of our experimental setup in section III. We review data processing, as implemented in our system, in section IV and provide the numerical results in section V. Finally, we conclude in section VI.

\section{Approach AND System Model}

\section{A The Radio link}

The unmodulated radio-frequency (RF) carrier transmitted by the hub can be written as

$$
s_{\mathrm{hub}}(t)=\sqrt{2 P_{\mathrm{c}}} \exp \left[j\left(\omega_{\mathrm{c}} t+\phi_{\mathrm{c}}\right)\right],
$$

where $P_{\mathrm{c}}$ is the transmitted RF power and $\omega_{\mathrm{c}}, \phi_{\mathrm{c}}$ are the angular frequency and phase of the transmitted RF carrier, respectively.

At the sensor, the received signal is simply reflected back out of the same antenna that received it. Specifically, the sensor modulates information by appropriately controlling a semiconductor device (e.g., a field-effect transistor (FET) or a diode) attached to the antenna, that varies the reflection coefficient. In practical implementations of backscatter, the reflection coefficient has only two distinct possible states and backscatter modulation is accomplished by alternating between these two states. For this reason, the reflection coefficient $\eta(t)$ can be approximated by:

$$
\eta(t)=\eta_{0} b(t)
$$

where $\eta_{0}$ is a constant and $b(t)$ is the modulating binary function controlled by each sensor that only takes the values \pm 1 .

Consequently, the signal backscattered from a single sensor and received at the hub can be written as:

$$
r_{\mathrm{hub}}(t)=\sqrt{2 P_{\mathrm{c}}} S_{\mathrm{L}}(t) \eta_{0} b(t) \exp \left(j \omega_{c} t+\phi_{0}(t)\right)
$$

where $S_{\mathrm{L}}(t)$ is the time-varying (due to non-constant propagation conditions) two-way (round-trip) signal amplitude loss, due to propagation from hub to sensor and back to hub (which also depends on gains of hub and sensor antennas, their distance and carrier wavelength). Alternatively,

$$
r_{\text {hub }}(t)=A(t) b(t) \exp \left(j \omega_{c} t+\phi_{0}(t)\right),
$$

where $A(t)$ is the (real) time-varying, due to multipath, amplitude and $\phi_{0}(t)$ is the phase of the received carrier signal, assumed uniformly distributed in the interval $[0,2 \pi)$. 


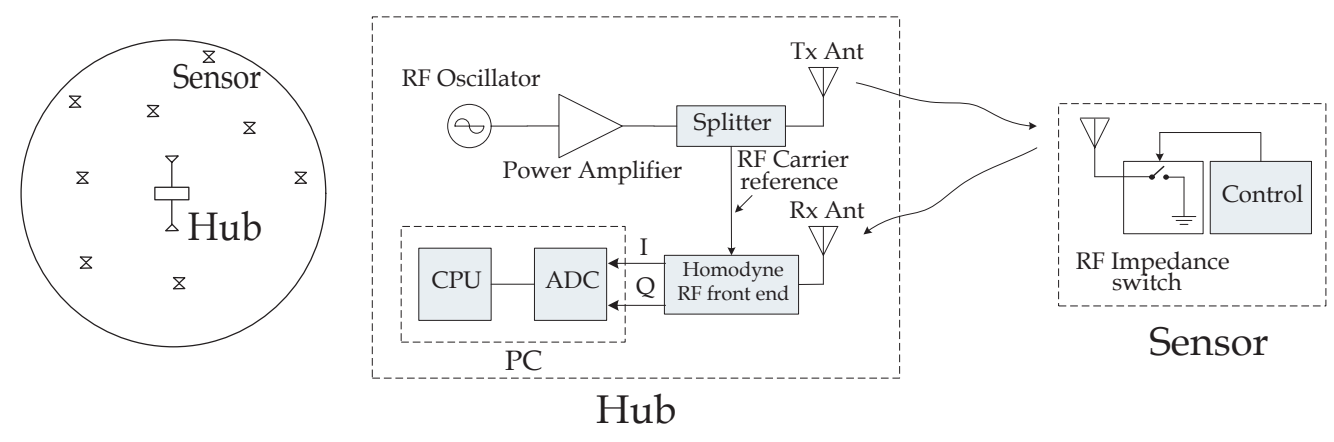

Figure 1: Backscatter radio between the hub and various ultra-low cost sensors and the main processing hardware and software blocks, at the hub and a single sensor. Notice that the transmitting element at each sensor, is simply a switch made of a transistor.

\section{B Sensors Access and Subcarrier Modulation}

Given that we are interested in ultra-low cost and simple sensors that backscatter continuously ${ }^{1}$, within approximately $100 \mathrm{~m}$ from the hub, simultaneous access of the wireless medium by all sensors becomes challenging. This is due to a) the absence of a receiver structure at each sensor and b) the continuous transmission of information by all sensors, which both exclude a plethora of anti-collision protocols theoretically analyzed in the RFID literature. The latter are based on carrier sense and collision detection (and thus require a receiver structure at each sensor) or assume that sensors share the medium (and thus, do not continuously transmit information).

We address the wireless access challenge, by appropriate subcarrier modulation of $b(t)$ at each sensor. Even though the carrier transmitted from the hub is common for all sensors, the modulating function $b(t)$ generated by the controller of each sensor is a square wave at a predetermined frequency, referred to as the subcarrier frequency and unique for each sensor. Subcarrier frequencies range between $67 \mathrm{kHz}$ to $200 \mathrm{kHz}$ and information from each sensor is modulated onto its (unique) subcarrier. Notice that $b(t)$ can take only two values $( \pm 1)$ and, therefore, binary modulation is the only option.

The allocation of different subcarrier frequencies among sensors, in principle explicitly addresses the access problem. In practice however, information on modulated subcarrier of one sensor could leak in as interference to other sensors operating in adjacent subcarrier frequencies, due to a) continuous backscattering operation of all sensors (i.e. switching on/off from control transistor) and b) lack of baseband filtering at each sensor. The amount of such interference, depends on the power spectrum of the chosen subcarrier modulation as well as the number of sensors. In order to minimize such interference, we chose a specific flavor of angle modulation, namely minimumshift keying (MSK). MSK is a special case of frequency-shift keying (FSK) with power spectrum $S_{\mathrm{MSK}}(f)$ that drops with the fourth power of frequency:

$$
S_{\mathrm{MSK}}(f) \approx \frac{1}{(5 T f)^{4}},
$$

where $T$ is the bit period, as opposed to other binary modu-

\footnotetext{
${ }^{1}$ Optimization of parameters relevant to backscattered power can be found in [2].
}

lation techniques such as binary pulse-amplitude modulation (BPAM) or binary phase shift keying (BPSK) where power spectrum drops as the second power of frequency [3].

We are interested in bit periods on the order of $T=100$ msec (or $10 \mathrm{bps}$ ) for each sensor, requiring a nominal bandwidth of $10 \mathrm{~Hz}$ for each sensor (and practically on the order of tens-of $\mathrm{Hz}$ ). Given that we are allocating $200-67=133$ $\mathrm{kHz}$ for all sensors, it reasonably to expect that careful subcarrier frequency allocation among $100-200$ sensors in that band can minimize interference, especially when MSK is utilized. In fact, it has been shown in [4] that even random (=not careful) subcarrier frequency allocation, for hundreds of sensors scattered in a $100 \mathrm{~m}$ radius do not compromise performance due to interference (collision) among sensors with MSK subcarrier modulation. Building on these result, and targeting for $10 \mathrm{bps}$ per sensor, for approx. 100 sensors in $100 \mathrm{~m}$ radius from hub (similarly to [4]), we can safely assume that our system operates in the noise-limited and not interference-limited regime. As such, noise thereinafter refers to thermal noise at the hub.

We note that from the perspective of modulation and access scheme, our system is radically different than that proposed in [5], where amplitude modulation was used to detect high bit-rate information from a single rapidly moving tag, or work in [6] where a simple receiver at each tag allowed individual interrogation of each tag from the reader (hub) at each time interval. Our system is designed to interrogate several, low-bit rate sensors continuously and simultaneously.

We write $b(t)$ as an expression of a square wave of (angular) frequency $\omega_{\mathrm{s}}$, with angle modulation represented by $\phi_{\mathrm{s}}(t)$. It is convenient to represent the square wave in terms of its Fourier components:

$$
b(t)=\frac{4}{\pi} \sum_{k=0}^{+\infty} \frac{1}{2 k+1} \cos \left[(2 k+1)\left(\omega_{\mathrm{s}} t+\phi_{\mathrm{s}}(t)\right)\right] .
$$

In our system, we employ digital FSK with no baseband filtering and the phase-modulation waveform can be analytically written as:

$$
\phi_{\mathrm{s}}(t)=2 \pi \Delta f_{s} \int_{0}^{t} \sum_{k} B[k] p(\tau-k T) d \tau,
$$

where $\Delta f_{s}$ is the frequency deviation, $B[k]= \pm 1$ is the sensor information bit pattern, $p(t)$ is a rectangular pulse of duration 
$T$ and amplitude 1 , and $T$ is the bit duration. As already mentioned, typical values of $T$ are in the range of $100 \mathrm{~ms}$, corresponding to a bit-rate of $10 \mathrm{bps}$. A typical value for $\Delta f_{s}$ is $\Delta f_{s}=0.25 / T$, corresponding to MSK modulation. Thus, the bandwidth of the sensor signal is rather narrow and, especially, much narrower than the available subcarrier frequency range of $67 \mathrm{kHz}-200 \mathrm{kHz}$. This is the key to our system's effectiveness as a simple, low-cost solution.

Our implemented receiver at the hub front-end only sees the fundamental component of the square wave, in order to simplify receiver design: to capture higher harmonics, the receiver would need a much wider bandwidth, but we see from (6) that the fundamental component holds $\approx 80 \%$ of the total power of the square wave. Thus, including all the harmonics would, at best, improve signal strength by about $1 \mathrm{~dB}$. This does not justify the substantial additional cost and complexity associated with the wider bandwidth.

If we substitute the fundamental term from (6) into (4), we obtain the following expression for the backscatter signal at the hub, due to a single generic sensor with subcarrier angular frequency $\omega_{s}=2 \pi f_{s}$ :

$$
r_{\text {hub }}(t)=\frac{4}{\pi} A(t) \exp \left(j \omega_{c} t+\phi_{0}(t)\right) \cos \left(\omega_{s} t+\phi_{\mathrm{s}}(t)\right) .
$$

\section{Homodyne RF Front-End Processing}

It is common in backscatter communication to use homodyne detection in the receiver. This is particularly effective because the receiver is co-located with the source of the RF carrier and, by using the transmitted signal itself as a reference for homodyne detection, i) phase noise cancels out [7] and, ii) the signal is frequency-shifted to $0-\mathrm{Hz}$ center frequency with real and imaginary parts extracted. Thus, the output of the RF homodyne front-end is a pair of real signals:

$$
\left\{\begin{array}{c}
y_{\mathrm{I}}(t)=(4 / \pi) A(t) \cos \left(\phi_{0}(t)\right) \cos \left(\omega_{s} t+\phi_{\mathrm{S}}(t)\right) \\
y_{\mathrm{Q}}(t)=(4 / \pi) A(t) \sin \left(\phi_{0}(t)\right) \cos \left(\omega_{s} t+\phi_{\mathrm{s}}(t)\right)
\end{array}\right.
$$

where $y_{\mathrm{I}}(t), y_{\mathrm{Q}}(t)$ are the real part (the "in-phase" component) and the imaginary part (the "quadrature" component) of (8), respectively.

\section{Signal-to-Noise Ratio}

Up to now, we have ignored the presence of thermal noise at the hub receiver. In reality, the receiver picks up noise, in addition to the sensor signal. Also, a full system will include a plurality of sensors, each with its own subcarrier frequency. A complete representation of the received signal can be written as:

$$
r_{\mathrm{I}}(t)=\sum_{k=1}^{N_{\text {sns }}} y_{\mathrm{I} k}(t)+n_{\mathrm{I}}(t), r_{\mathrm{Q}}(t)=\sum_{k=1}^{N_{\text {sns }}} y_{\mathrm{Q} k}(t)+n_{\mathrm{Q}}(t),
$$

where $N_{\text {sns }}$ is the total number of sensors in the system, and $n_{\mathrm{I}}(t), n_{\mathrm{Q}}(t)$ represent additive, identical and independent gaussian noise components with variance $\sigma^{2}$ related to their power spectral density $N_{0}$ by:

$$
\sigma^{2}=\mathbb{E}\left\{n_{\mathrm{I}}^{2}\right\}=\mathbb{E}\left\{n_{\mathrm{Q}}^{2}\right\}=W N_{0},
$$

with cutoff frequency $W$ (receiver bandwidth), at least 200 $\mathrm{kHz}$.

If we denote the information bit period by $T_{\mathrm{i}} \geq T$ and the average energy per bit by $E_{\mathrm{b}}$, the SNR $\rho$ experienced by each sensor can be easily derived from (9):

$$
\rho=\frac{E_{\mathrm{b}}}{N_{0}}=\frac{8}{\pi^{2}} \frac{A^{2} T_{\mathrm{i}}}{\sigma^{2} / W} .
$$

We note that the above formula assumes that the two signal components of eq. (9) are combined coherently (optimally), as explained subsequently in eq. (13). Moreover, the above formula implies that the amplitude of the received signal remains constant, in order for the SNR calculation to be meaningful. That is why the dependence on time has be dropped $(A(t) \equiv A)$. We discuss this again in section $\mathrm{V}$.

\section{EXPERIMENTAL SYSTEM OVERVIEW}

Fig. 1 shows block diagrams of the hub and sensors used in our experimental system. The frequency of the transmitted RF carrier is tunable in the range $900-930 \mathrm{MHz}$. Transmitted power level is $5 \mathrm{~mW}$. In-phase (I) and quadrature (Q) signal components are digitized by a pair of 16-bit Analog-to-Digital Converters (ADC). The sampling rate is $1 \mathrm{MHz}$ for each waveform. With this sampling rate, the Nyquist frequency is $500 \mathrm{kHz}$, which allows subcarrier frequencies up to $200 \mathrm{kHz}$ to be accurately recorded with enough margin for digital filtering of adjacent signals. The 16-bit resolution allows adequate dynamic range to accommodate sensors at various distances from the hub.

Because the subcarriers generated by the sensors are square pulses, they will have a strong third-harmonic component. Therefore, if the maximum possible subcarrier frequency is $200 \mathrm{kHz}$, the minimum usable subcarrier frequency will be $200 / 3 \simeq 67 \mathrm{kHz}$; otherwise, the third harmonic of the lowfrequency sensors interferes with higher-frequency sensors. So, with these parameters, we have about $133 \mathrm{kHz}$ of bandwidth available for sensor subcarriers, which is sufficient to support several hundred sensors [4].

The sensor is designed around a low-power, coin batteryequipped microcontroller (TI MSP430) driving a low-power RF switch. Battery is only used to power the microcontroller which implements backscatter radio by simply switching on/off one transistor. In that way, sensor circuitry is not powered by the field transmitted by the hub and thus, backscatter radio operates in extended ranges. ${ }^{2}$ The microcontroller clock is derived from a low-cost watch-type crystal at $32768 \mathrm{~Hz}$ with an accuracy of $100 \mathrm{ppm}$. The specific subcarrier frequency for each sensor is produced by a software-based phase-locked-loop (PLL), which makes MSK modulation easy to implement.

\section{DATA PRocessing}

After the homodyne RF front-end processing, the signal before the ADC is modeled by eqs. (9)-(10). That is the signal sampled at $1 \mathrm{MHz}$. The purpose of data processing is to extract the

\footnotetext{
${ }^{2}$ We note that our system architecture can be applied in passive (batteryless) sensors as well.
} 
useful narrowband signals, around the subcarrier frequencies of all sensors. At the same time, we need to reduce the sampling rate appropriately, from $1 \mathrm{MHz}$ to a few tens of $\mathrm{Hz}$. The latter becomes important when a large number of sensor signals (on the order of 100-1000) requires processing, and therefore, memory and processing time per sensor signal cannot be unbounded in practical realizations. Due to space constraints, we briefly describe the techniques followed in our implementation. Details will be reported elsewhere.

\section{A Signal Acquisition}

The first required task is to identify how many sensors are operating and which are their respective (subcarrier) frequencies. Our receiver first calculates the average power spectrum using efficient fast fourier transforms (FFT) and then performs a search for signals with bandwidth close to the desired (a few tens of $\mathrm{Hz}$ ), taking into account the accuracy of the utilized sensor crystals.

After the estimation of the subcarrier frequency $f_{s}=\omega_{s} / 2 \pi$ of each sensor, our system performs signal filtering around the subcarrier frequency (for each sensor) and downsampling from $1 \mathrm{MHz}$ to $100 \mathrm{~Hz}$. In that way, we donwconvert the received signal to dc and at the same time, we reduce the number of samples/sec/sensor by a factor of $10^{4}$, vastly decreasing the computation burden.

Filtering around the subcarrier frequency $\omega_{s}$ is performed using the impulse response that coincides with the nominal pulse of MSK modulation:

$$
h(t)= \begin{cases}\cos \left(\frac{\pi}{2 T} t\right) & -T \leq t \leq T \\ 0 & \text { elsewhere }\end{cases}
$$

Filtering is performed at the frequency domain representation of $y_{\mathrm{I}}(t)+j y_{\mathrm{Q}}(t)$ in two stages, extracting two signals for each subcarrier frequency $f_{s}$ (and sensor). The first signal is extracted after multiplication with $H\left(f-f_{s}\right)$ (where $H(f)$ is the frequency response of $h(t))$ and thus, corresponds to the positive-frequencies component. Similarly we extract the second signal that corresponds to the negative-frequencies component. Filtering for both signals is done by exploiting efficient FFT techniques.

The rationale behind using positive and negative subcarrier frequency signal components, is provided in the following section. We emphasize the fact that downsampling is also performed during this filtering stage.

\section{B Signal Combining and Extraction for the BackScatter Channel}

As mentioned, the SNR expression in (11) assumes that the two detected signals, $r_{\mathrm{I}}(t)$ and $r_{\mathrm{Q}}(t)$ are combined optimally, using maximal-ratio (coherent) combining. Our case is a rather simple instance, where optimal combining simply corresponds to rotating the (complex) received signal by $-\phi_{0}$ and detecting the result along the real axis. Specifically, the optimal combination of $r_{\mathrm{I}}(t)$ and $r_{\mathrm{Q}}(t)$ is

$$
r_{\mathrm{opt}}(t)=\cos \phi_{0} r_{\mathrm{I}}(t)+\sin \phi_{0} r_{\mathrm{Q}}(t)
$$

We see that, in order to perform coherent combining of the two received components, the receiver needs to know the value of $\phi_{0}$. This is not easy to achieve in practice, as $\phi_{0}$ is the phase of the RF carrier in the received signal which depends on many factors, including multi-path. The reason why it is difficult for the receiver to estimate $\phi_{0} \equiv \phi_{0}(t)$ has to do with the low bit-rate (about $10 \mathrm{bps}$ ). Factors affecting $\phi_{0}$ (such as multipath) cannot be assumed to be sufficient stable, over multiple bit periods for the receiver to obtain an accurate estimate of $\phi_{0}$. In fact, one can safely assume that for 10 bps (or equivalently $T=100 \mathrm{msec}$ ), $\phi_{0}$ can change within a single bit period.

This is a distinctive characteristic in our backscatter communication system, given the extended duration of the bit interval (or equivalently, the low-bit rate for each sensor). Therefore, estimation of $\phi_{0}$ is not feasible and thus, we cannot implement coherent combining of $r_{\mathrm{I}}(t)$ and $r_{\mathrm{Q}}(t)$ in our system. Without the option of such combining, an important part of our work has been to identify combining techniques that are simple to implement but also with a reasonable performance penalty compared to coherent combining.

We write the complex baseband signal of (9) as:

$$
\begin{aligned}
y_{\mathrm{I}}(t)+j y_{\mathrm{Q}}(t)= & \frac{2 A(t)}{\pi}\left\{\exp \left(j\left(\phi_{0}(t)+\phi_{\mathrm{s}}(t)\right)\right) \exp \left(j \omega_{\mathrm{s}} t\right)+\right. \\
& \left.+\exp \left(j\left(\phi_{0}(t)-\phi_{\mathrm{s}}(t)\right)\right) \exp \left(-j \omega_{\mathrm{s}} t\right)\right\} \\
= & y_{+}(t) \exp \left(+j \omega_{\mathrm{s}} t\right)+y_{-}(t) \exp \left(-j \omega_{\mathrm{s}} t\right),
\end{aligned}
$$

with

$$
\left\{\begin{array}{l}
y_{+}(t)=(2 A(t) / \pi) \exp \left[j\left(\phi_{0}(t)+\phi_{\mathrm{s}}(t)\right)\right] \\
y_{-}(t)=(2 A(t) / \pi) \exp \left[j\left(\phi_{0}(t)-\phi_{\mathrm{s}}(t)\right)\right]
\end{array} .\right.
$$

The presence of $\phi_{0}(t)$ in (14) precludes (optimal) coherent combining of $y_{+}(t)$ and $y_{-}(t)$. This is not surprising because the manipulations we applied to obtain (14) from (9) are a form of coordinate rotation; however the format of (14) suggests a nonlinear combining technique that makes $\phi_{0}(t)$ disappear. Specifically, if we multiply $y_{+}(t)$ by the complex conjugate of $y_{-}(t)$, we obtain

$$
\Lambda(t)=y_{+}(t) y_{-}^{*}(t)=\frac{4 A^{2}(t)}{\pi^{2}} \exp \left(2 j \phi_{\mathrm{s}}(t)\right)
$$

which does not include $\phi_{0}$.

We observe that $\Lambda(t)$ is still an angle-modulated waveform, but the angle modulation is doubled. In our system, we extract the message bit sequence $B(t)$ by implementing in software a detector of the instantaneous frequency:

$$
B(t)=\frac{1}{4 \pi \Delta f_{s}} \frac{d}{d t}<\Lambda(t)
$$

where the operator $<x(t)$ denotes phase of complex signal $x(t)$. The factor of 4 (as opposed to a factor of 2) is due to the signal combining technique followed in (16), where phase is doubled. We note that despite of the inherent non-linearity of the technique, performance was reasonable, as shown in section $\mathrm{V}$. 


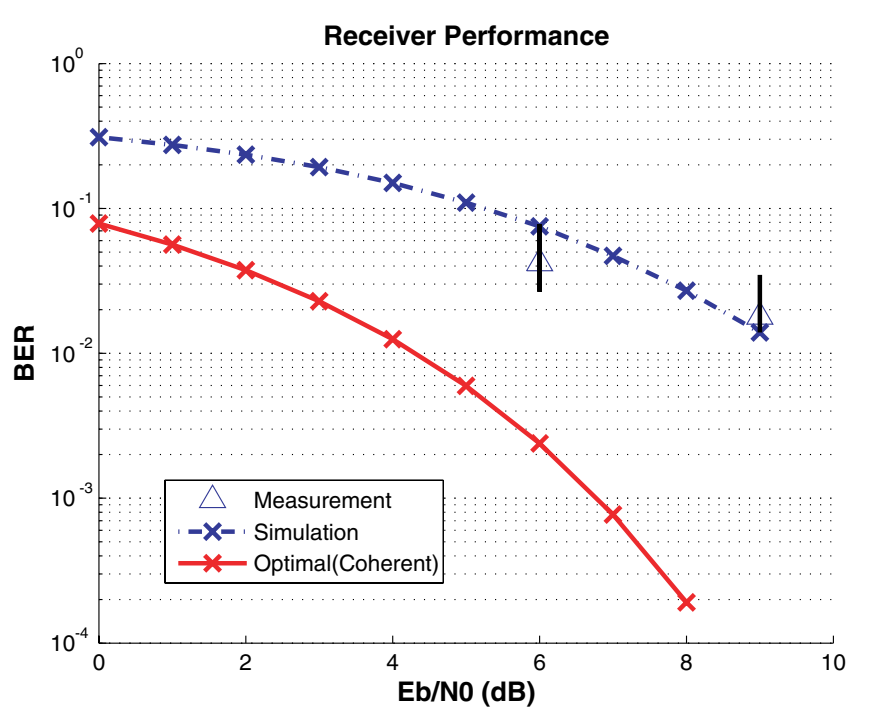

Figure 2: Simulation and experimentation results with our hardware setup. In the latter case, we also plot measurement error standard deviation bars.

\section{RESULTS}

We evaluated performance of our system, using both simulation as well as experimental measurements with our hardware/software prototype. Given that sensors operate in different subcarrier frequencies and thus, interference among them is not an issue (as discussed previously, in section II.B), we focus on bit-error-rate (BER) results from a single sensor.

Fig. 2 provides simulation results, assuming a sensor backscattering a unique word of 128 bits (for message synchronization) and a message of another 128 bits. The plot includes the overall BER of our system (including detection and synchronization) and contrasts it to the theoretically optimal, based on coherent detection (with perfect synchronization). We remark that the latter is practically infeasible given the slow bit rate in our system, as explained before, in section IV.B. The overall performance of our system (including synchronization as well as detection) provided an approximately $5-\mathrm{dB}$ performance loss, compared to the theoretically optimal coherent detection (with perfect synchronization). For a wireless backscatter link of distance $d$ between hub and sensor, assuming power loss proportional ${ }^{3}$ to $1 / d^{8}$, increase of $5 \mathrm{~dB}$ in signal power provides approx. $15 \%$ increase in distance. ${ }^{4}$

Fig. 2 also provides experimental results using our prototype system for 2 specific SNR values. The first is at $6 \mathrm{~dB}$ and the second is at $9 \mathrm{~dB}$. The measurements were acquired overnight at two different locations, within approximately 15 meters from the interrogator hub, in a typical indoor office space environment (with many scatterers and attenuators due to building columns, glass separators etc). We expect such range to be further extended when we increase the transmission power to the

\footnotetext{
${ }^{3}$ Taking into account reflection from the ground, one way propagation provides signal power loss proportionally to $1 / d^{4}$ and thus, round-trip power los becomes proportional to $1 / d^{8}$.

${ }^{4}$ Maximum range of 100 meters outdoors is the final goal.
}

maximum level of approximately 1 Watt while operating our system outdoors, away from the RF-cluttering environment of our lab.

BER measurements on the actual prototype were performed overnight so as to minimize multipath due to people movement inside the building. In that way, SNR measured on the received signal alongside eq. (11), was accurate and stable. The same techniques used for SNR measurement on the actual signal, was also tested and validated in simulated signal (where SNR was a priori known) both at the passband signal (at $1 \mathrm{MHz}$ sampling rate), as well as on the baseband, downsampled and filtered signal.

Finally, fig. 2 includes measurement error bars, assuming a Poisson distribution for the number of bit errors. Average number of bit errors and variance were calculated by the product of bit error rate (calculated from simulation) with total number of bits used in the experimental measurement. The measurement error bars have length that corresponds to 2 standard deviations. We observe that our acquired measurements were within the error bars, demonstrating that our end-to-end implementation (including down-conversion, down-sampling, synchronization and detection) met the theoretical error requirements.

\section{CONCLUSiON}

We have implemented a system for simultaneous reception of signals continuously radio-backscattered from several low-cost sensors. We hope this work sparks interest in the fertile are of backscatter communication, especially suited for low-bit rate, low-power and ultra-low cost sensor networks.

\section{ACKNOWLEDGEMENTS}

The authors would like to thank Dr. Joe Marks, Dr. Joseph Katz, Dr. Bill Yerazunis and Dr. Paul Dietz, for their insightful technical comments throughout this work.

\section{REFERENCES}

[1] K. Finkenzeller, RFID Handbook: Fundamentals and Applications in Contactless Smart Cards and Identification, 2nd ed. Wiley, New York, 2003.

[2] K. Rao, P. V. Nikitin, and S. F. Lam, "Antenna Design for UHF RFID Tags: A Review and a Practical Application," IEEE Trans. Antennas Propagat., vol. 52, no. 12, Dec. 2005.

[3] S. Benedetto, E. Biglieri, and V. Castellani, Digital Transmission Theory. Prentice-Hall, New Jersey, 1987.

[4] G. Vannucci, D. Leigh, and J. Katz, "Wide-area Long-Range Unidirectional Sensor (WALRUS) Network," in Proceedings of the IEEE International Conference on Mobile Ad hoc and Sensor Systems, Nov. 2005.

[5] A. R. Koelle, S. W. Depp, and R. W. Freyman, "Short-Range RadioTelemetry for Electronic Identification, using Modulated RF Backscatter," Proc. IEEE, pp. 1260-1261, Aug. 1975.

[6] J. Curty, N. Joehl, C. Dehollain, and M. J. Declercq, "Remotely Powered Addressable UHF RFID Integrated System," IEEE J. Solid-State Circuits, vol. 40, no. 11, Nov. 2005.

[7] B. Razavi, RF Microelectronics. Prentice-Hall, New Jersey, 1998. 